\title{
Enhancing Qos Using a Novel Task Scheduling Approach in Cloud Computing
}

\author{
Sudan Jha \\ School of Computer Engineering, Kalinga Institute of Industrial Technology, Odisha, India, \\ jhasudan@hotmail.com
}

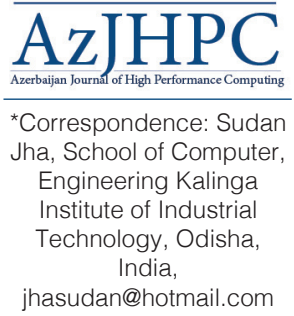

Abstract

Customers' satisfaction at the ensured organizations has a strong reliance on the specific execution of appropriated registering from the perspectives of benefit bit and undertaking booking. Disregarding the way that this sort of organizations don't require customers' need of information about the particular detail of advantages (e.g., a machine), in light of the way that authority communities (e.g., specialists or server ranches) have arranged, virtualized and interfaced the benefits with the objective that the customers are awed that an earth-shattering machine has just relegated them. Everything considered, fulfilling the assurances determinedly depends upon the specific execution of circulated figuring, especially on the ideal approach to passing on an obliged measure of cloud resources for an enormous variety of or even unlimited errands requested by customers that much of the time ask for speedy organization yet minimal effort. For a reason for depiction in the field of appropriated registering, an errand is as often as possible named a Cloud or isolated into more diminutive Clouds [1] [2] [3], and an advantage is frequently conveyed as a VM. This paper is, thusly, induced to enhance the procedures of benefit assignment and errand booking by altogether considering the collections and capabilities of the characteristics and features of Clouds and machines. Midway as a result of different potential attractions of circulated registering, confident promoters defeat to ensure customers in a huge variety of organizations in a large and often, and the ensures as often as possible stable more than adequate.

Keywords: cloud computing, software, software testing, software complexities, securities, task scheduling and their attributes (TSAT)

\section{Introduction}

By the day's end, customers' satisfaction at the ensured organizations has a strong reliance on the specific execution of appropriated figuring from the 
perspectives of benefit assignment and undertaking arranging notwithstanding the way that this sort of organizations don't require customers' learning of the particular detail of advantages (e.g., a machine), in light of the way that the advantages have been cheerful virtualized and interfaced by authority centers (e.g., delegates or server ranches) with the objective that the customers are propelled that an extreme machine exclusively apportioned them. Everything considered, fulfilling the assurances decidedly depends upon the specific execution of disseminated figuring, especially on the ideal approach to passing on a limited measure of cloud advantages for a great grouping of or even endless endeavors requested by customers that frequently ask for snappy organization yet minimal effort. For a reason for depiction in the field of conveyed figuring, a task has consistently delegated a Cloud or isolated into more diminutive Clouds [1] [2] [3], and a benefit is as often as possible imparted as a VM. This paper is, along these lines, propelled to enhance the strategies for resource bit and task booking by liberally pondering the combinations and capabilities of the qualities and features of Clouds and machines. Not entirely in light of different potential attractions of appropriated registering, confident promoters are uncommonly defeated to ensure customers a great grouping of organizations, and the ensures every now and again stable more than adequate. Dynamic, the combinations and refinements of the characteristics and features of Clouds and machines have been rarely investigated for the VM assignment and Cloud booking, to the best learning of this paper author. Circulated registering addresses a rapidly creating design in the IT industry and it is progressing to empower customers to share giant figuring resources over frameworks to achieve overpowering and complex data taking care of in a cloud circumstance. Moreover, it may give some engaging organizations, for instance, the well - alluded to pay-as-you-go mode that customers can buy handling organizations correctly when the organizations are required, as opposed to buying and keeping up an expensive machine that isn't routinely used.

Objectives: - Predictable with the inspiration, the general target of this paper is to bargain the examination shortage by coordinating distinctive Clouds with various VMs in view of various properties and dynamic workload status of assets, in order to enhance the nature of administration for distributed computing from the points of view of undertaking booking and asset designation. To approach the general goal, the accompanying sub-targets and methodologies are arranged in a wellordered manner: (1) Introduce and broaden the idea of characteristic and after that plan a VM portion and Cloud booking system equipped for sifting the qualities and measure of lining Clouds for ideal VM utilization. The errands can be separated into various gatherings as indicated by their natural or appointed characteristics, for example, stockpiling concentrated, calculation serious, GPU assets, and high-payfor-need, i.e., the traits can be progressively organized or especially characterized by client or supplier's inclination and changing the status of the cloud condition.

Contribution: - Assess and study the execution change in VM apportioning and Cloud booking by using Task Scheduling and their attributes (TSAT), in spite of the way that the evaluation relies upon a revised interpretation of TSAT including a LoT that for the most part channels differing attributes and a HiT that is thoroughly 
screening particular characteristics. Upgrade the sensibility between different Clouds and assorted machines through using the TSAT system to viably assign the most suitable advantages for the assignments with relative properties.

\section{Literature review}

There are additionally definitions concentrating on the structure and constitution of mists [8]:" Cloud processing is characterized as a sort of parallel and dispersed framework comprising of a gathering of between associated and virtualized PCs that rare powerfully provisioned and displayed as at least one bound together figuring assets in light of understandings, built up through a transaction between the specialist co-op and purchasers".

Another definition that is frequently referred to underscores the "administration" given by the distributed computing to people. In general [6]: "Distributed computing is the conveyance of processing as an administration as opposed to an item, whereby shared assets, programming, and data are given to PCs and different gadgets as an utility (like the power lattice) over a system (regularly the Internet)." Some definitions consider the evolvement history and contrast from other figuring innovations. The accompanying articulation mirrors [7] says "Distributed computing is created from Grid registering and it consolidates the conventional ideas of Grid Computing and distributed computing. For instance, NIST proposed the accompanying definition that features the "model" essentialness of distributed computing. This is broadly alluded to in the distributed computing network [5]: "Distributed computing is a model for empowering omnipresent, advantageous, on-request arrange access to a mutual pool of configurable figuring assets (e.g., systems,) that can be quickly provisioned and discharged with insignificant administration exertion or specialist organization cooperation. Reference [10] says that regardless of the current contrasts in definition, they a recognized that distributed computing may convey the favorable circumstances or advantages to both individual and business clients. Scalability: This is the capacity of a framework to deal with a developing measure of work, or its capability to be extended keeping in mind the end goal to suit that development. With the advance of distributed computing, clients including individual and business clients can use or keep up the online assets.

Besides, the compensation per-utilization model of distributed computing empowers end clients to pay for the assets in the cloud when required, as opposed to making any speculation on assets. Clients can rapidly scale up or down the assets in distributed computing as indicated by their requirements without owning the assets as their property.

Gao, Bai \& Tsai (2011) provides a comprehensive tutorial on cloud testing and cloud-based application testing. They provide clear concepts, discusses the individual objectives, features, requirements, and needs in cloud testing. They suggested that in future more innovative testing techniques and solutions, and quality of services standards are needed to support on-demand testing services in a scalable cloud infrastructure.

Ashraf, Rauf \& Mahmood (2012) proposed an algorithm, which prioritizes 
the system test cases based on the six factors customer priority, changes in requirement, implementation complexity, requirement traceability, execution time and fault impact of requirement.

Brara \& Maitra (2012) suggested a rough formulation for the reliability of software in a cloud-computing environment. They included a formula is just an idea, and it can be improvised further to include other aspects featuring cloud computing. They substantiated by pieces of evidence in the form of test trials and study of statistical trends thereof to arrive at refinement of the proposed formula to cater to all types of cloud computing situations. They suggested that in future Companies should pilot cloud-based testing as early as they feel comfortable to ensure they gain insights and benefits as these service offerings mature and become mainstream. therefore, organizations seriously considering deploying testing as a service need an experienced partner that can ensure cost reductions are achieved or exceeded over time and that time-to-market of software products or services

Incki, Ari \& Sozar (2012) provided an overview of the main contributions, trends, gaps, opportunities, challenges, and possible research directions. They provided a review of software testing over the cloud literature and categorize the body of work in the field. They presented a classification of current research studies, identified gaps in the literature and investigated the correlation of software testing with different deployment models of cloud computing.

Katherine \& Alagarsamy (2012) investigates the software testing in a cloud platform which includes cloud testing models, recent research work, commercial tools, and research issues. Testing software requires enough resources and budget to complete it successfully. Compared with conventional testing methods, cloud testing emphasizes more on system testing and online testing. This approach is due to the novel design and development methods imposed by cloud computing.

Prakash \& Gopalakrishanan (2012) focused on the challenges faced by companies in moving to a cloud environment, concerning security, reliability, and manageability, which the organizations should focus on only through rigorous testing. They explained the benefits of cloud computing and move towards the testing challenges faced by testers.

Priyanka, Chana \& Rana (2012) described the systematic mapping process, discussed the results of the mapping study and threats to the validity of the study. The systematic mapping process was described regarding the research questions defined, searching keywords used, the exclusion and inclusion criteria. The results of the study were classified into several categories and analyzed. They show the areas of research within Cloud-based testing that have been done by answering the questions that were defined initially. They presented results from a systematic review of empirical evaluations of cloud-based testing techniques.

\section{Proposed model}

As of now existing asset booking and allotment procedures talked about in the past part of writing survey are for the most part concentrating on assignment sum, 
and there have been different examinations on planning system that depends on coordinating the assortment and multifaceted nature of clients' errands and server farms' assets. By perusing characteristics of each Cloud, errand planning applies two-level property sifting and edges [35] on a lining framework that will in the long run ideally organize the handling of assignments having distinctive qualities and diminish execution time of the Clouds. This paper endeavors to characterize a standard for sorting various properties and influence two-level to trait definition, and applies a two-level sifted property coordinating into lining framework for organizing asset preparing request. For relieving the examination shortage of properties, this printed material considers both load qualities and sum in the proposed calculation for ideal assignment booking. Along these lines, other assignment booking and asset distribution systems that have considered undertaking need would then be able to be coordinated with the TSAT procedure proposed in this paper. The characteristics of solicitations from clients should be considered in the assignment booking and asset portion. A la mode, there was no standard definition portraying characteristics of assignments or occupations, and different examinations address this issue, to the best learning of the paper creator. For instance, although planning routine is regularly made out of three stages of asset spotting, asset inclination, and undertaking accommodation [34], the three stages are mostly in light of the measure of lining loads. Choosing an ideal hub to execute an assignment as indicated by its asset prerequisites and the wellness between asset hubs and undertakings may enhance errand execution and asset use productivity.

\subsection{Model to use various Attributes into a Cloud}

In this section, a technique for grouping and classification of credits is proposed to render the qualities dependably go with assignment substance of a Cloud to give accommodation to coordinating distinctive employments for various assets. At the point when a Cloud of this write is gotten, the perusiCloudng can effortlessly peruse the traits of the Cloud, or decoding convention consented to and recognized by cloud dealer or server farm, without exasperating the substance of the Cloud. Solicitations from various clients may relate distinctive asset requests: a few workloads might be CPU-concentrated though others are capacity - escalated, and some of them may even require an overwhelming utilization of exceptional equipment like GPUs to accomplish emotional execution picks up [36]. Generally, characteristics got through breaking down the employment, since clients present a vocation to an asset with a couple of insights, including memory prerequisite, capacity to utilize exceptional highlights like GPUs, and the due date, if necessary [36]. Note that several qualities could likewise be dispersed in numerous different segments of the Cloud to enhance the distinguishing proof of the traits or even be figured first and the set in the Cloud to improve the security or the protection of the properties. Figure1 outlines a streamlined structure alternative of a Cloud, wherein diverse characteristics are masterminded in a bordering segment. Different setups of machines are accessible in the cloud with various limits and execution, and cloud suppliers additionally like promoting and offering their uniqueness and contrasts of their mists and administrations, either from specialized or valuing the viewpoint. 


\subsection{Cloud Consisting of Both Content and Attributes}

The technique proposed in this paper is posting all the fundamental characteristics of a Cloud and joining the qualities into a Cloud. On the off chance that each kind of machine is organized to offer the most reasonable undertakings or convey a decent cost-execution and exchange off for the activity, the execution of cloud might be productively enhanced, e.g., demands are prepared all the more effective and assets are utilized all the more monetarily. For instance, web application asks for the most part concern more about reaction time, and group occupations regularly require being done before the due date and consequently concern the measure of CPU time, and RAM apportioned to the employment. Notwithstanding the assortment and many-sided quality of solicitations, assets in distributed computing condition are additionally very different and not uniform in numerous viewpoints, i.e., assets are innately heterogeneous in the cloud. Be that as it may, characterization of qualities is extremely intricate as indicated by various prerequisite. The benefits of collection or grouping of occupations help with arranging indistinguishable employments asking for similar assets, in like manner the asset can be provisioned [37].

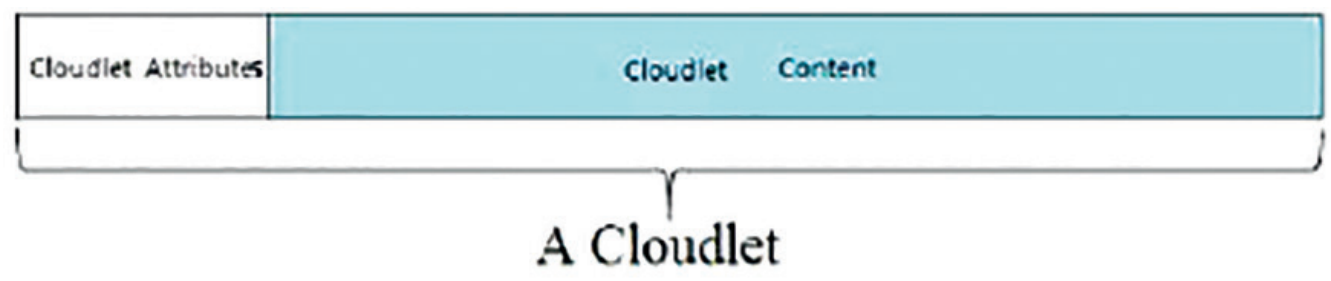

Figure 1 : A Cloud consisting of contiguous attribute part and content part

\subsection{Concept of TSAT}

This is moreover to help ensure a cloudlet can be coordinated a VM; (2) The credit edge is to ensure that the Clouds with remarkable characteristics (e.g., high need is apportioned to the properties) have the advantage to be planned to the best sensible resource if various Clouds with various qualities are requesting a comparable resource. If the VMs are not possessed, simply unforgiving isolating is performed so the Clouds with various credits can be allowed to enter the holding up the line until the point that the moment that as far as possible is wholly included. As the line size may be dynamic, so once the master finds cut down edge is accomplished, by then strict isolating is constrained on all requesting and just picked endeavors are allowed to enter the holding up the line between the lower and maximum breaking points, until the point that the upper edge comes. Toward the day's end, when a cloudlet is allowed to enter the holding up a line of a VM and meets up at the VM, it is dealt with instantly if the VM is free; for the most part, the cloudlet remains in the holding up line someplace before the VM. Right, when the furthest reaches of a VM is accomplished; the holding up a line of the VM recognizes no more requests. 
Table 1. Examples of attributes based on different level of threshold

\begin{tabular}{ll} 
Below lower threshold & Between lower and higher thresholds \\
\hline computation-intensive & Deadline far or near \\
\hline storage-intensive & Military or civil \\
\hline GPU-intensive & High pay or low pay \\
\hline
\end{tabular}

For engineering, appropriate Clouds to an available resource (e.g., storage space or enlisting unit), an essential option for the plan of characteristics can be a two-level filtering framework

- A lower level isolating (level 1) and upper-level filtering (level 2) of attributes. In SRPT, the accompanying occupation to be served is the one with the tiniest leftover getting ready timelining.

- The machine for dealing can snatch both of the upper or lower edges. In the real cloud condition, a considerable measure of VMs exist, so it would be a noteworthy and complex work to choose the extent of each breaking point for each exceptional VM.

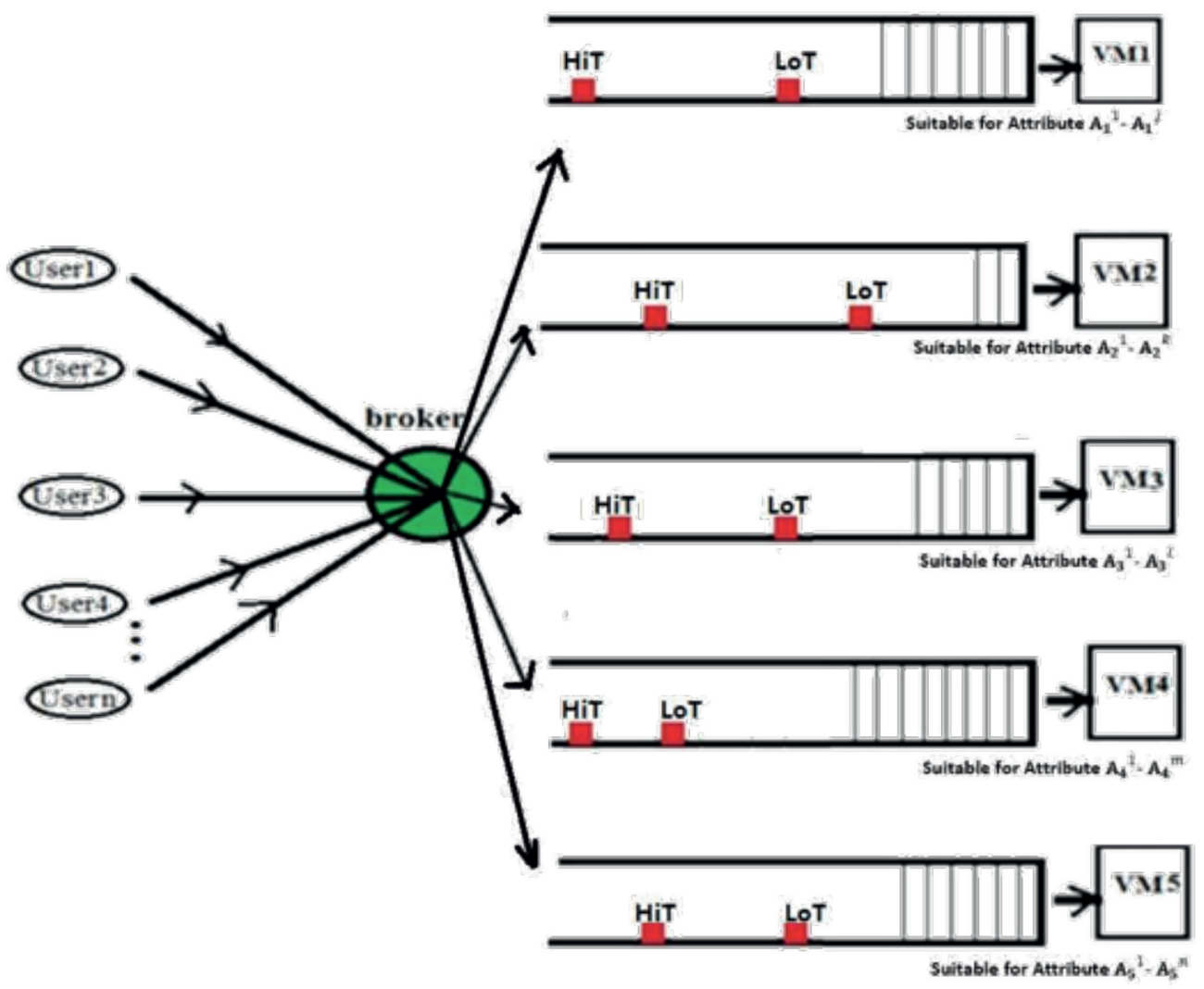

Figure 2 (a): Awareness of TSAT 
When it is fused with TSAT, the task with the smallest size coating under both of the upper or lower points of confinement can be gotten by the machine for getting ready. The lower edge plays out an unforgiving filtering of characteristics and the more lifted sum edge powers a strict isolating on coming Clouds. In the TSAT computation, each VM has a tight sitting line for moving toward Clouds before the Clouds are set up by the VM. The degree of upper and lower edges are picked by VM's capacity, CPU speed, and some extraordinary components. For straightforwardness, it is controlled that no cloudlet is ousted from the holding up line once it has been allowed to enter the line, disregarding the way that the cloudlet may have a lower requirement than a cloudlet coming after it. The inspiration driving this paper is to take a gander at the endeavor arranging and resource apportioning execution of TSAT, so only an unraveled decision is favored for the feasibility to inspect, that is, same characteristics are set for the two furthest reaches of each and every VM.

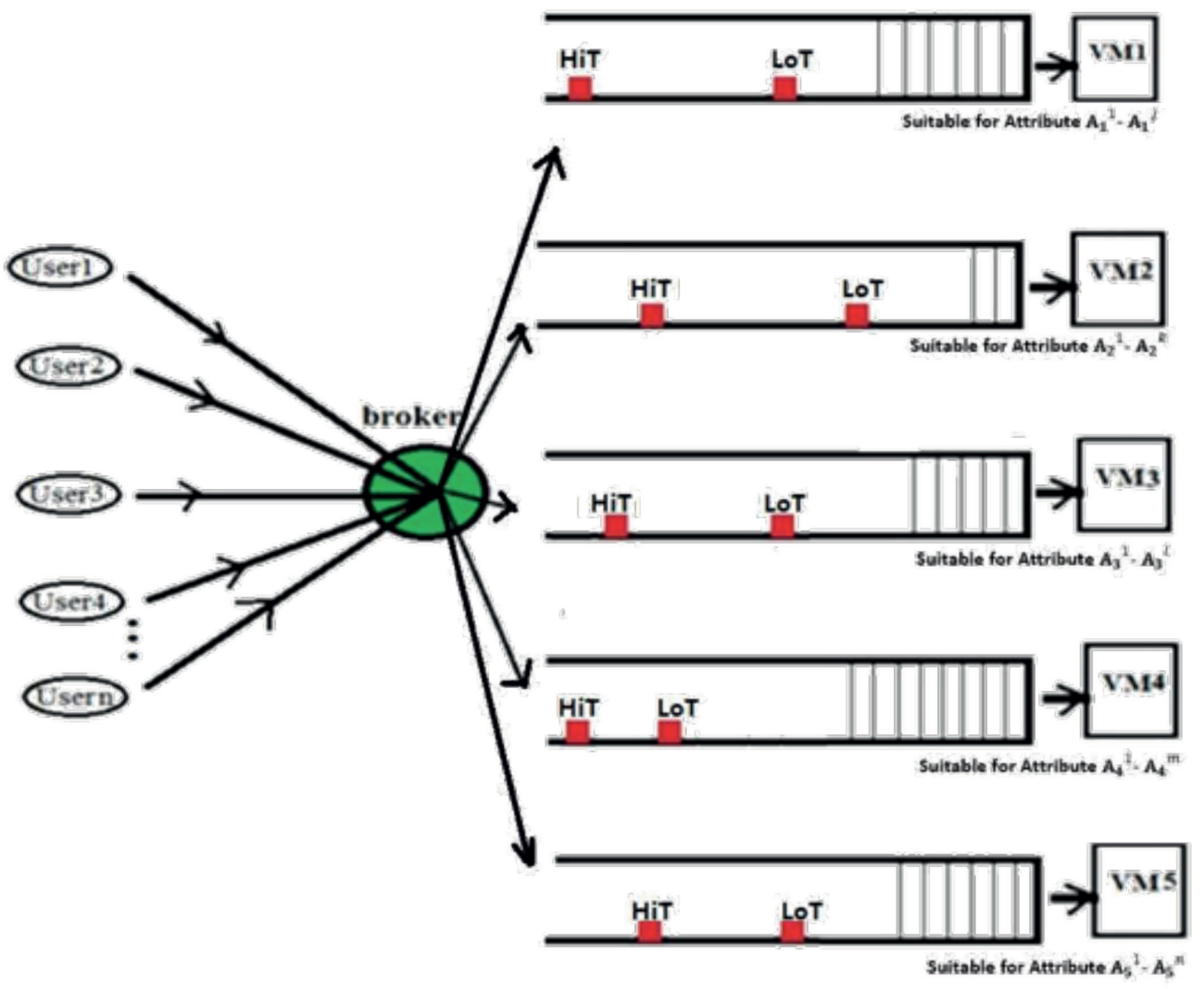

Figure 2 (b): A simplified special awareness case of TSAT 
Overall, the edge degree of each VM may be particular because of various gear and programming setups and capacities. Figure 2 (a) speaks to a general schematic of different edge enormity for each exceptional VM. Since each time a VM takes in one cloudlet for getting ready, so once the treatment of the cloudlet has been done, another cloudlet straightforwardly after the cloudlet is recognized just into the VM. Figure 2 (b) exhibits an enhanced shape with the same edges regards for every novel VM. So underneath the lower level edge, there are a more significant number of sorts of attributes than among lower and furthest points of confinement.

The holding up line includes two fragments made by the lower and upper edges, as appeared in Figure 2 (a) (b). Two fundamentals for the assurance could be taken after: (1) as far as possible is to evade a VM to be overload. For example, a cloudlet with the summit due date that is sincerely moving closer may be sorted out a higher need than various endeavors with expelled culmination course of occasions, and military errands may have a higher need than regular assignments. The TSAT strategy can moreover be meant TSAT count, i.e., to pick the best proper resource (VM) for each cloudlet, composed attributes and workload entirety are used as assurance parameters.

Table 2. Notes for Figures 2 (a) and (b)

\begin{tabular}{cc}
\hline VM1 & More suitable for cloudlets with attributes A - A \\
\hline VM2 & More suitable for cloudlets with attributes A - A \\
\hline VM3 & More suitable for cloudlets with attributes A - A \\
\hline VM4 & More suitable for cloudlets with attributes A - A \\
\hline VM5 & More suitable for cloudlets with attributes A - A \\
\hline HiT & Upper Threshold \\
\hline
\end{tabular}

\subsection{Filtering the Tasks@ LowerThreshold}

Once the line of a VM for figuring is accomplishing the full covering utmost of the VM's lower edge, by then the higher edge of the same VM is started. In the next step coming Clouds requesting estimation is filtered more altogether, e.g., additional relative "high pay" is added to its parental "count raised" credit to shape a "high pay + computation concentrated" channel. An instance of setting the property standard for the unpalatable channel of the lower level farthest point could be the basic or assessed sorts of Clouds rather than quick and dirty subtypes, identifying with classes of parental and relative properties. Figure 3 (a) addresses a general occurrence of different cutoff estimate for each unprecedented VM and Figure 3 (b) addresses an improved case that all remarkable VMs have a comparative most extraordinary motivation for all 
lower edges, and Table 3 delineates the properties of VMs that are fitting for to help clear up figures 3 (a) and (b). For instance, "requesting limit" and "figuring concentrated" could be two basic creates or parental attributes, and "limit of budgetary data", "amassing of photo", and "limit of video" could be three subtypes or relative properties under the basic kind or parental property of "requesting limit". Under as far as possible, the Clouds are facilitated to different VMs just in light of the maternal characteristics rather than the relative properties.

Table 3. Notes for Figures 3(a) and (b)

VM1 (under Lower threshold) Cloudlets with Attribute A - A

VM2(under Lower threshold) Cloudlets with Attribute A - A

VM3(under Lower threshold) Cloudlets with Attribute A - A

\begin{tabular}{cl} 
VM4(under Lower threshold) & Cloudlets with Attribute A - A \\
\hline VM5(under Lower threshold) & Cloudlets with Attribute A - A \\
\hline LoT & Lower Threshold \\
\hline
\end{tabular}

Right, when line appraises is under lower confine, it suggests there are not very many customer assignments, i.e., Clouds, requesting the utilization of a VM, so the VM is reasonably free. For example, for a VM that is sensible for Clouds with nature of requiring computation, all the estimation raised assignments is recognized by the VM, paying little regard to its completing due date or pay for the organization. As a result, late coming "low pay + count raised" Clouds may be facilitated to the line of another VM for figuring to apportion the best resource of the server homestead to the Clouds with most astonishing need (high pay is an instance of expanding most vital need). By the day's end, other restricting properties, for instance, a due date close or far and high pay or low would then have the capacity to be disregarded at this level, and these characteristics are commonly considered and sorted out when there are various errands past the extent of as far as possible. As for the parental trademark "estimation raised," its relative characteristics could be "High pay computation" and "Iow pay count." By the day's end, the brutal channel standard can be bright for necessary sorts of Clouds, yet darkened for discretionary sorts that have a place with a first sort. Was observed that this course of action, for the most part, helps to illuminate the channel of attributes at different levels of edges. This paper won't look 
at much about how to portray the wording of characteristics, as this requires a generously more full examination - for example, those genuine estimation Clouds that are requesting count would be composed to those VMs that are more sensible for figuring, paying little notice to high pay or low pay.

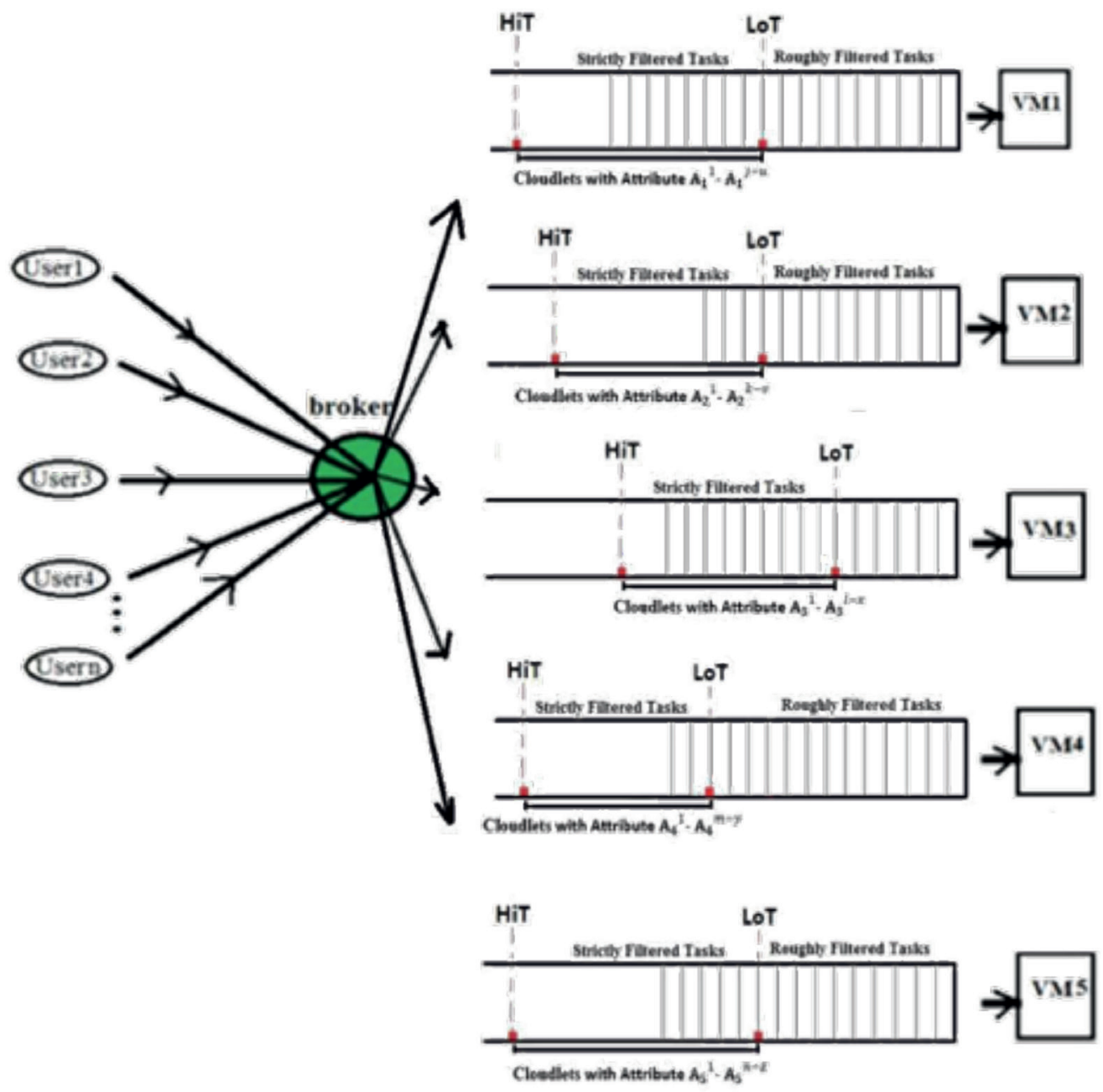

Figure 3 (a): Strictly filtering tasks between low and high Thresholds 


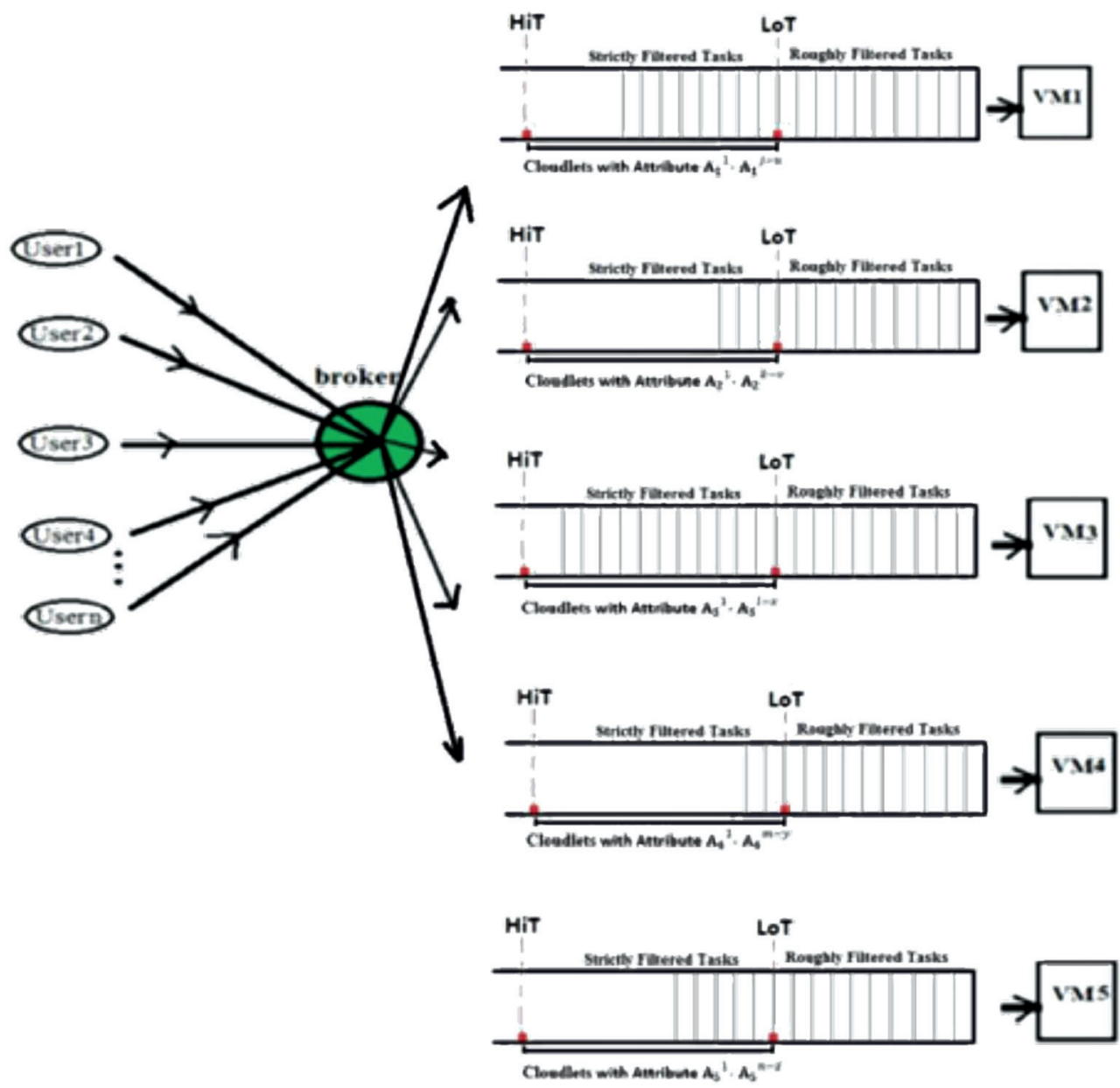

Figure 3 (b): Strictly filtered tasks between low and high threshold

\section{Implementation}

This section picks a cloud diversion gadget to duplicate a bona fide cloud condition keeping in mind the end goal to test the execution of task arranging and resource parcel with the proposed TSAT methodology before familiarizing it with the honest to goodness cloud condition. The tool compartment supports both structures and leads exhibiting of cloud system parts, for instance, server ranches, VMs (VMs) and resource provisioning game plans [21].

4.1. Introduction to CloudSim was at first made in GRIDS investigate focus at the University of Melbourne, which has been viewed as an ordinary cloud 
reenactment toolkit that licenses exhibiting and entertainment of and moreover investigating different roads concerning, appropriated processing circumstances [3]. After entirely examining the code and stream of Cloudsim, this paper laid out the chain of significance and process schematically, as spoke to in Figure 4 The technique begins with a customer request, and this request is taken as a commitment by the cloud system and named as a cloudlet in CloudSim. Genuine substances or classes consolidated into CloudSim gadget are displayed as takes after [3]: Datacenter go-between: it is made as a center out of the street between cloud customers and providers to pass on benefits over the fogs. An organization pro addressing a client checks resource information through a component called Cloud Information Service (CIS), chooses sensible resources and after that shows the request to the picked resources for planning. In any case, no such a schematic portrayal has been represented, regardless of the way that Cloudsim has been gotten by a couple of specialists for the propagation of different parts of disseminated processing. A schematic depiction for the levels of leadership and technique of how Cloudsim reenacts the plan of cloud organizations to clients over the framework is outstandingly helpful for us to have a significant picture point of view of a cloud. Starting late, CloudSim has been by and large used for surveying diverse figurings including resource allocation, provisioning, and making arrangements for the late year, and has been wound up being a successful reenactment toolkit. Cloud Information Service (CIS): this is a substance that gives organizations, for instance, cloud resource enlistment, requesting and disclosure. We can observe that the Cloudsim has given most components or classes to amass a cloud area, which in this manner can furthermore give the going with different sorts of functionalities [3]. Fortunately, as shown in the past composing outline, some cloud amusement gadgets have been made to test cloud execution. In the midst of the advantage decision and settlement methodology, specific benefit task and undertaking booking game plans are used for guiding customer sales to the fitting VMs planned with CPU, memory, storing and exchange speed. Support for showing and amusement of considerable scale disseminated figuring server ranches. In any case, it is over the top and improbable to test an adolescent strategy that has not been composed in an honest to goodness cloud condition. The cloud resource table or summary (have listed, VM list) teaches their status to process Clouds by selecting themselves. While using CloudSim features, planners or investigators don't need to consider the lower level unobtrusive components of the cloudbased establishment. Notwithstanding whether the TSAT approach proposed in the past part would in every way that matters upgrade the execution of cloud requires tests in an honest to goodness cloud condition. Incoming about sections, the certain substances, and functionalities of CloudSim and moreover advantage giving method is displayed, and after that, the detailed stream graph and pseudocode of TSAT framework are introduced. Datacenter: it contains and models gear and programming organizations in the cloud which is administered by cloud expert associations. 


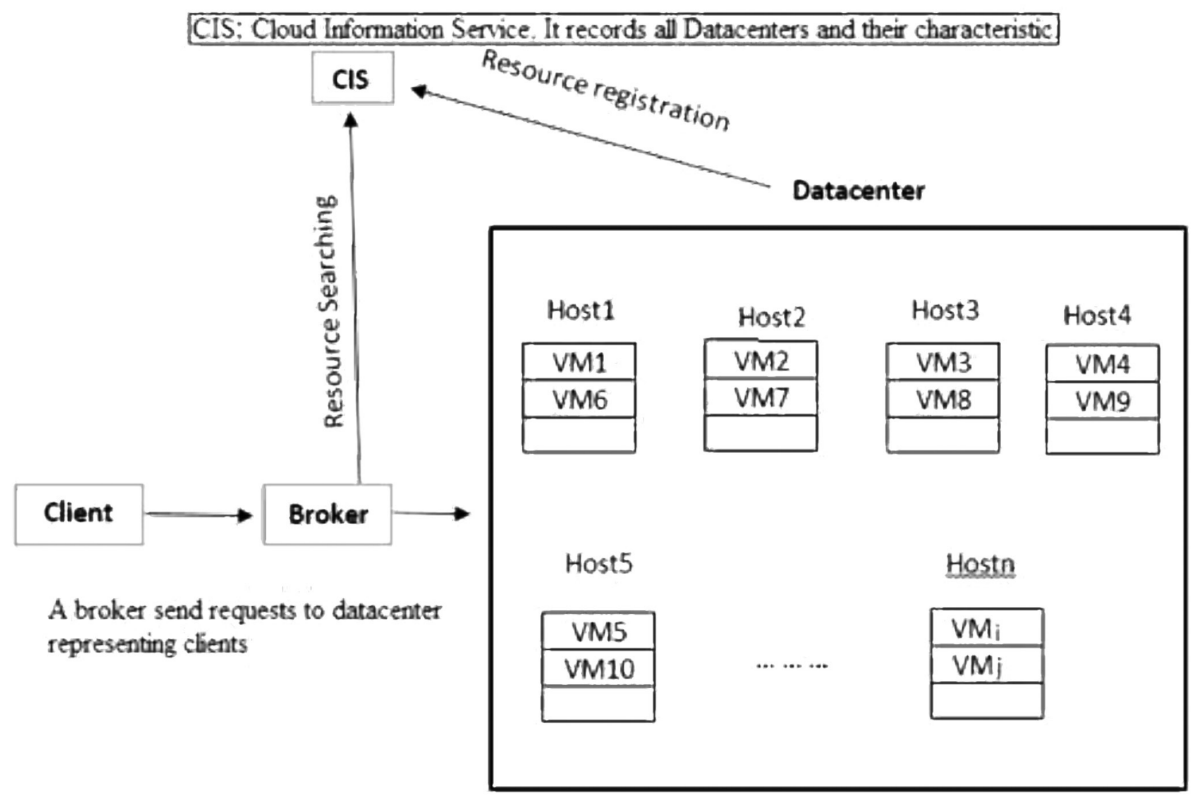

Figure 4: Hierarch y of simulating services to clients in CloudSim

\subsection{Extending CloudSim}

To execute the proposed TSAT calculation, we have to consider stretching out Cloudsim to incorporate TSAT from the three perspectives: Create Clouds, and VM Clusters Apply lower and upper limits on holding up the line of each VM to progressively recognize the heap measure of each VM. Because of the cloudlet class and VM groups, along these lines, coordinating Clouds with VM has checked whether attribute1 of Clouds coordinate VM's trademark. To discover VM gatherings to be coordinated for each cloudlet because of harsh property sifting, the code that performs both sum and characteristic separating is embedded into the specialist class. Select a most reasonable VM for each cloudlet and submit, through contrasting properties of Clouds and the reasonableness of each VM, and additionally through identifying the heap sum data of each VM. The merchant checks if a cloudlet trait matches with the appropriateness of an asset, and after that in like manner choose a fitting VM group for the cloudlet. For achieving the best proficiency in the execution of each undertaking, assignments are coordinated with VM groups when the merchant gets new demands from clients. For the examinations, this paper utilizes two writes to portray assignments and besides VM bunches: calculation, stockpiling. 
4.2.1. Create Clouds and VM Clusters:

The errand asked for by client may have diverse properties; hence some particular assets are required. This situation incorporates looking at and doles out credits to both Clouds and VMs (VMs) as indicated by their sorts and qualities.

Step 1: A basic illustration is adding two credit fields to cloudlet class: 'characteristic 1' and 'property 2', and trait 1 can be utilized for bringing down limit, and quality 2 can be utilized for upper edge. As to allocating credits to VMs, they can be characterized and gathered in light of various attributes, for example, calculation and capacity.

Step2: Create Clouds that have two distinct qualities.

Step 3: Because of generally coordinate, entirely coordinating require property 2 meet a few prerequisites, for example, close deadline or high pay.

Step 4: Match Clouds with VM groups.

Table 4. An example of the process of roughly filtering clusters for the matching

Cloudlet.JD

cloudlett: cloudlet100

cloudlet101-cloudlet20Q
Roughly Matched VM groups

vm1 1 vm3

vm2 2 vm4

The Proposed Algorithm finally is depicted as below: -

\section{A cloudlet joins the queue (producer) :}

Step 1: Initial load_status as 'OK into LoT'.

Step 2: A new cloudlet joins the queue.

Step 3: Check current queue size. If the current queue size is greater than or equal to LoT, update load status of the virtual machine to 'LoT FULL'; if the current size is greater than or equal to HiT update load status of the virtual machine to 'FULL'. Step 4: Repeat Step 1. 


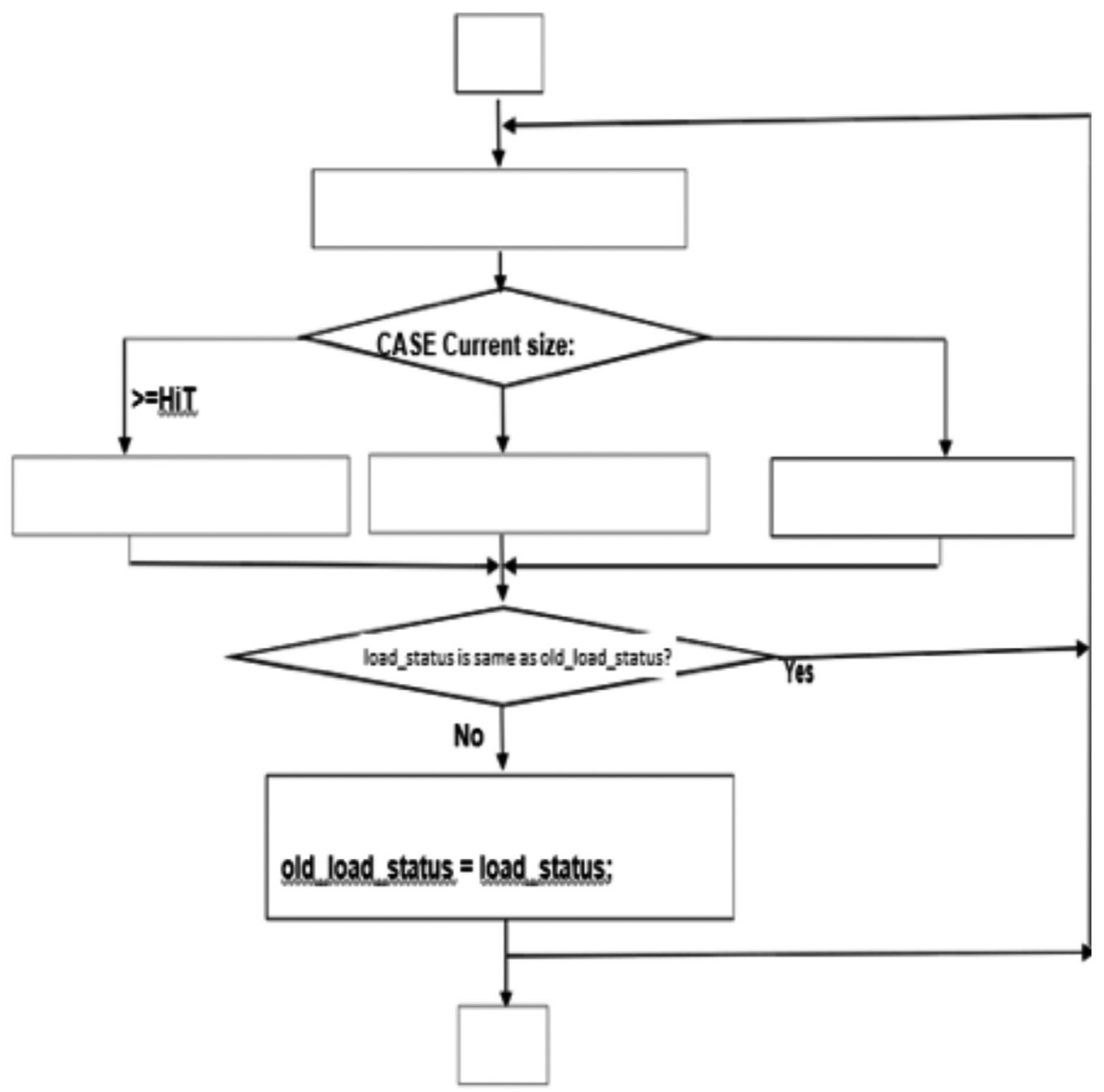

Figure 5 (a): Flowchart for the proposed algorithm

In the occasion that stack status is 'Package FULL', by then simply the Clouds that meet the strict channel standard are sent to the line of the VM, the Clouds which don't meet the strict channel standard is conveyed to another VM that may not be to a great degree sensible yet rather can do the action. If load status is 'okay into LoT,' by then, the delegate organizes the Clouds that meet the upsetting channel standard to this VM. 4.2.3 VM Selection and Cloudlet Submission Allocating an appropriate VM to Clouds relies upon stack status of VMs and characteristics of Clouds, and the codes are implanted into agent class. Each time before cloudlet convenience, the trader checks the load status (OK into LoT, LOT FULL, or HiT FULL) of VM. Distribute Clouds to the 


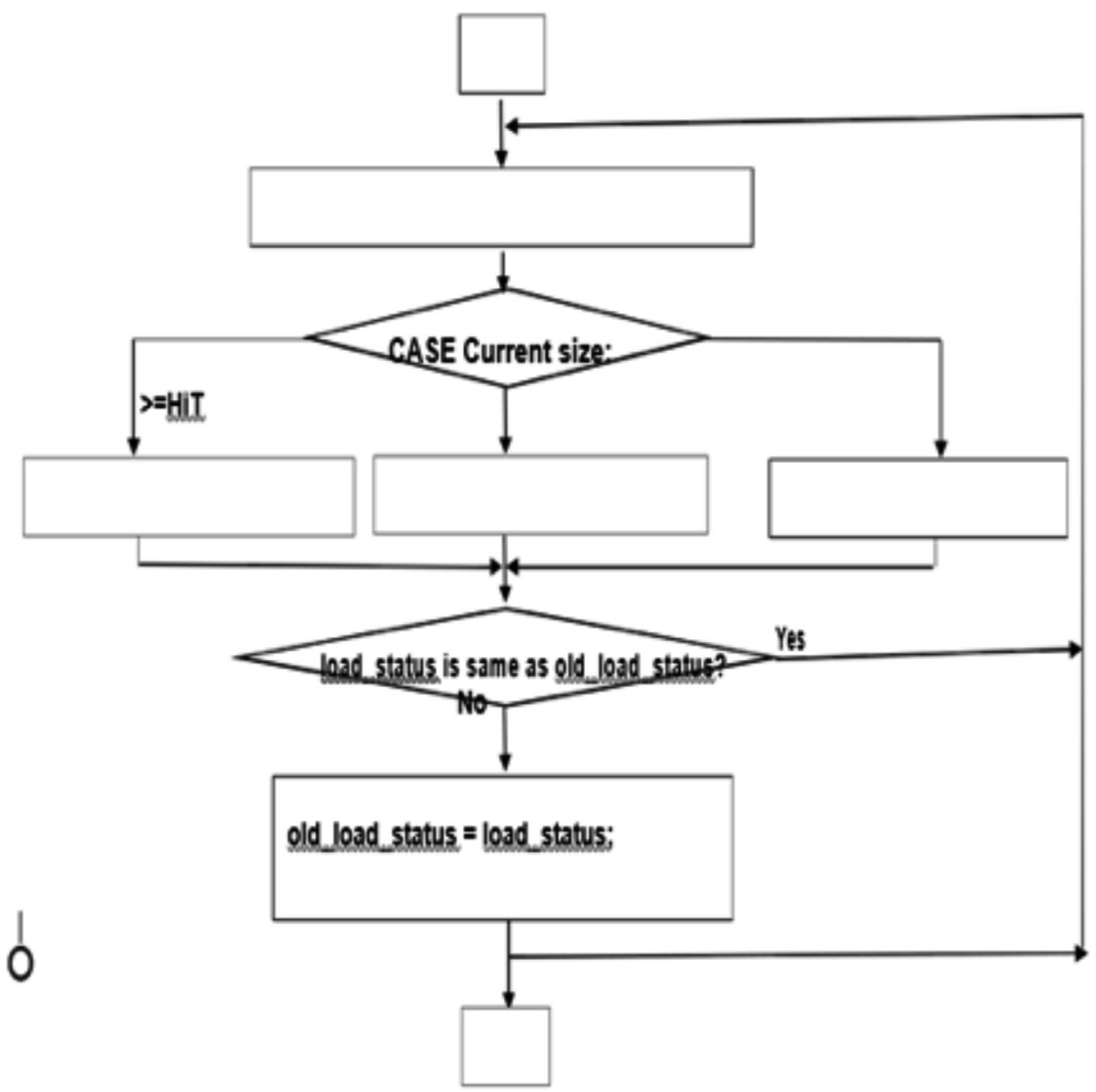

Figure 5 (b): Flowchart showing "Cloud queuing and the VM"

most sensible VM if the VM is available; (2) If the best proper VM is full, by then apportion the Clouds to a can-do VM, because a lower execution of the getting ready is better than not being taken care.

The final Algorithm

Output: submit (true) or not (false)

Boolean Function SendCloudletToVM(Cloudlet cloudlet)

$\left\{{ }^{*}\right.$ Find a roughly matched VM that is under LoT */ for (all VMs which is roughly matched)\{ 


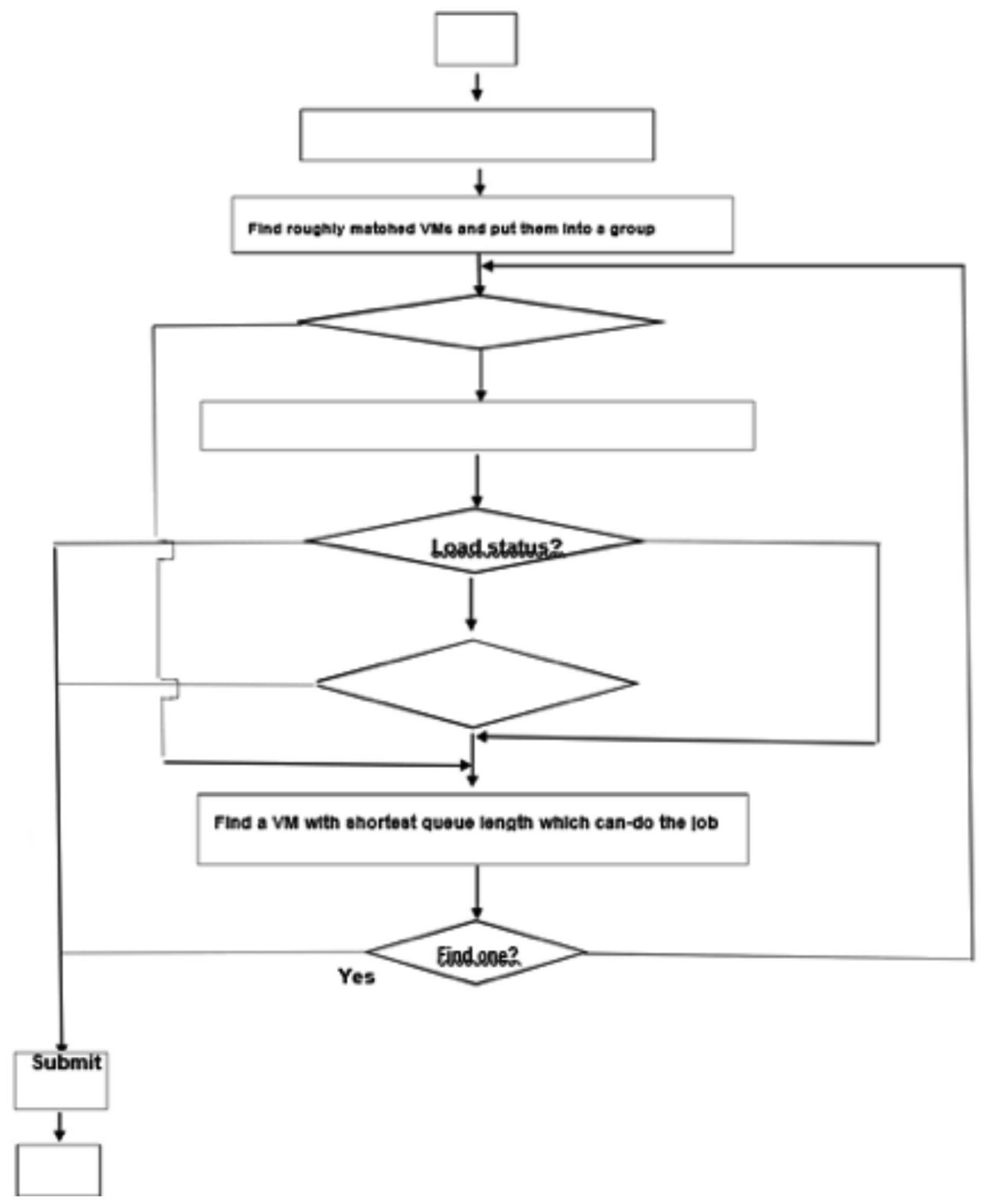

Figure 6: Flowchart showing "VM selection based on Amount and Attribute"

if (VM is "OK into LoT") \{ Submit cloudlet to the VM; \} Return true; 
${ }^{*}$ If all roughly matched VMs exceed LoT, find a strictly matched VM which is between LoT and HiT

for (all VMs which is roughly matched) \{

if (VM is "LoT FULL" and "strictly match") \{

Submit cloudlet to the VM;

\}) Return true;

$I^{*}$ If filtered out by the last two steps, find a VM which has the most slight load and can do the job */ for (all other VMs that can-do)\{

Find a VM with shortest queue length \{

Submit cloudlet to the VM;

\} $\quad$ Return true;

Return false;

\subsection{Process of Task Scheduling and Resource Allocation}

To represent the dynamic filling and lining of the lower limit and additionally the actuation of the higher edge, VM1 is taken for instance, and the dynamic occasions are amplified in Figure 8 After enacting of the VM1's higher edge, entirely separate late coming Clouds for being acknowledged into the line of VM1, so just the Clouds enumerated 18, 20, 23, 24, .. , 34, 69 can be orchestrated to the line of VM1 among the aggregate 90 unique kinds of Clouds, because lone these Clouds coordinate the strict sifting standard of VM1's higher limit. This is the reason in spite of the fact that VM1 is best appropriate for cloudlet 3 (e.g., with "high pay + calculation serious" traits), different Clouds (e.g., "Iow pay + calculation escalated") are likewise permitted to enter the line of VM1, in light of the fact that the Clouds all offer the same parental quality (e.g., calculation concentrated) that can be utilized for a harsh sifting by the lower edge. For an extremely bustling server farm, VMs' lower edges might be completely possessed by their particularly reasonable Clouds, Figure 7 (b) shows this circumstance: the Clouds are sifted into the line of each VM between the VM's lower and upper limits. Since the full filling of a lower limit is dynamic, i.e., when some Clouds are coming into the line of a VM, some different Clouds are vanishing in the line since they have been prepared by the VM. Figure 7 (a) demonstrates the circumstance in which every VM are still under lower edges because of the aggregate sum of Clouds (from first to No. 


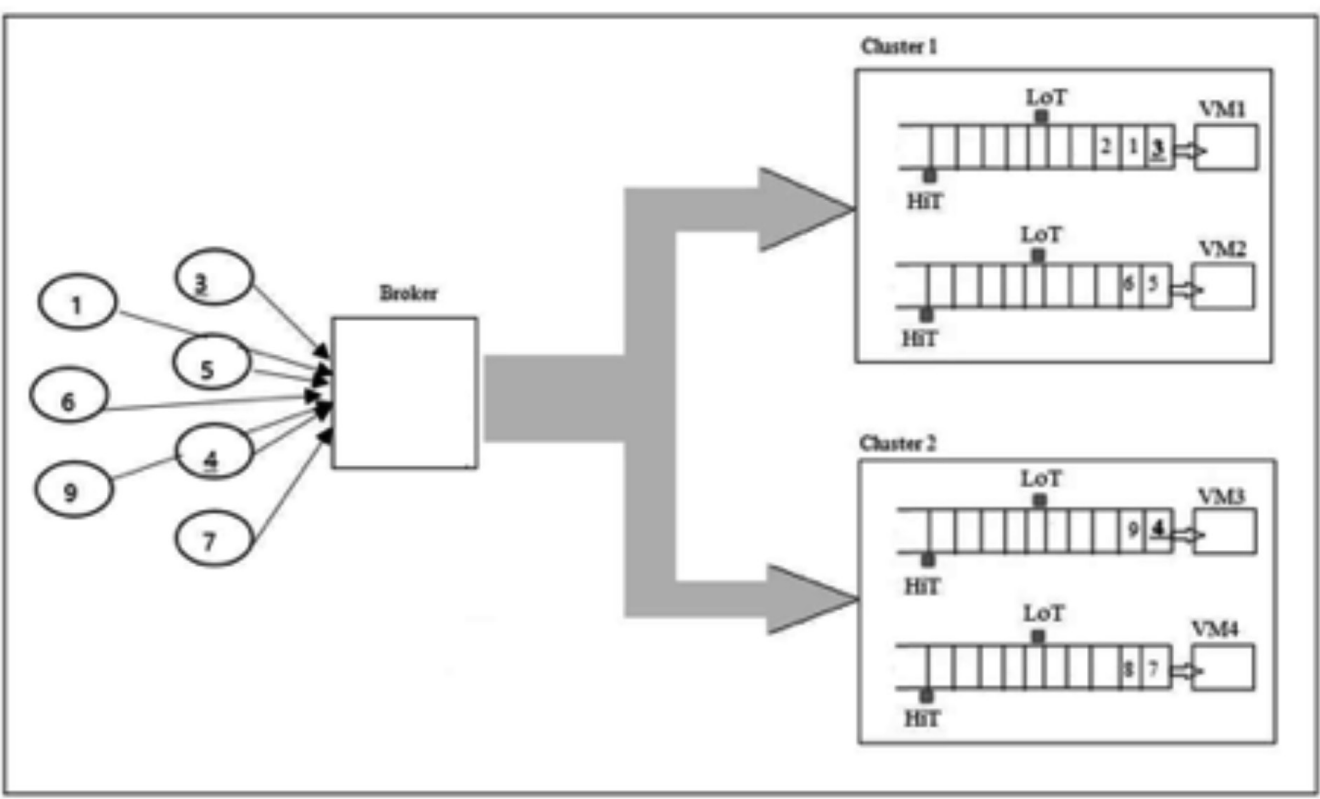

Figure 7 (a): Jobs Distribution

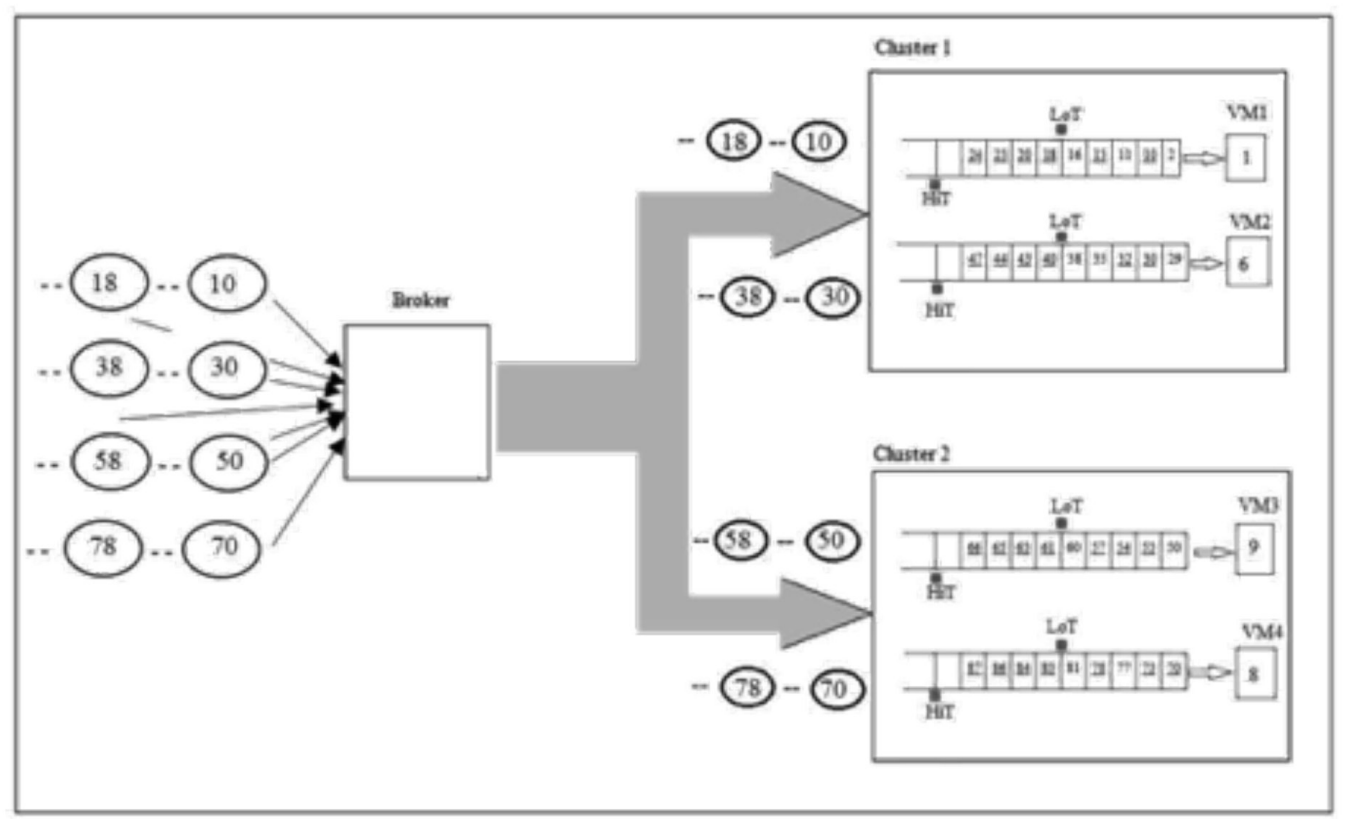

Figure 7 (b): Jobs Distribution based on Attribute and Thresholds 
To this end, this area shows the methodology how assignments are sent from the intermediary to VMs in Figures 7 (a) and (b), by utilizing a predetermined number of Clouds and VMs, despite the fact that there are substantially more Clouds and VMs in a genuine cloud condition for errand booking and asset designation. In this circumstance, its stricter channel is forced on approaching Clouds, i.e., a higher edge plays out this channel work. Note that the underlined Clouds in Figure 7 (a) and (b) are the Clouds that are more reasonable for the VM than different Clouds in a similar line of the VM. Ninth Clouds) is little, so the Clouds are just generally separated by the lower edges of the VMs. The conveyance of Clouds in light of traits and limits is likewise delineated in Figures 7 (a) and (b).

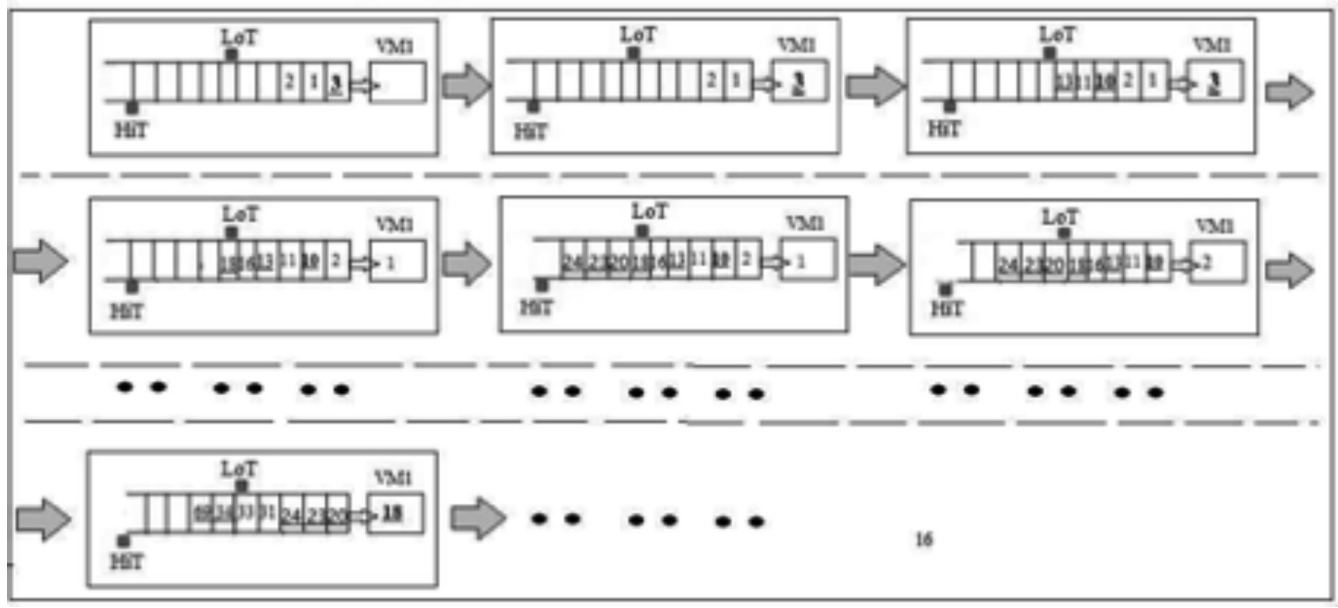

Figure 8. Process of excessive cloud entering the queue and filtered by upper threshold of VM1

\section{Experimental results and discussions}

\subsection{Performance Evaluation for proposed Algorithm}

In detail, at whatever point a cloudlet (I) is recognized by a VM (VM), if there were the same Clouds to be consigned to that VM already, by then the starting time of the cloudlet $(\mathrm{I})$ is zero. The starting time of the cloudlet is portrayed as the finishing time of the past cloudlet $(\mathrm{I}-1)$ on that VM.

Execution Time (Exec): It is the time length that is portrayed as the extent of $\mathrm{MI}$ (Million Instructions) constituting the cloudlet being set up to the MIPS (Million Instructions Per Second) of the circulated VM.

$\mathrm{ST}(\mathrm{i})=0$; [if $\mathrm{VM}(\mathrm{j})$ is in free] ST(i) > 0; [otherwise, $\mathrm{ST}(\mathrm{i})=$ finishing time of cloudlet(i-1)] .................................. (5.1) Acceptance rate: It is the extent of the measure of Clouds extremely recognized by a VM to the total whole of Clouds that are required to be recognized by the VM.

Landing Interval (AI): It implies the extent of the total time to the complete whole of Clouds that have met up at the server cultivate inside the total time. 
Table 5. Parameters used in the experiments of a data center

\begin{tabular}{|c|c|c|c|}
\hline \multicolumn{3}{|c|}{ Parameters reflecting attributes } & \multirow[b]{2}{*}{3000 mips } \\
\hline \multirow{2}{*}{\multicolumn{2}{|c|}{ (best for com putation) }} & CPU speed & \\
\hline & & & \\
\hline & & Storage & $50 G B$ \\
\hline \multirow{2}{*}{\multicolumn{2}{|c|}{ (best for storage) }} & CPU speed & 1000 mips \\
\hline & & Storage & $1000 \mathrm{~GB}$ \\
\hline \multirow{2}{*}{\multicolumn{2}{|c|}{$\begin{array}{c}\text { VM3 and VM4 } \\
\text { ( acceptable for } \\
\text { computation and storage) }\end{array}$}} & CPU speed & 2000 mips \\
\hline & & Storage & $300 \mathrm{~GB}$ \\
\hline \multirow{3}{*}{\multicolumn{2}{|c|}{ dimensions of thresholds }} & LoT for all VMs & 500 cloudlets \\
\hline & & Between HIT and LoT for all & 300 cloudlets \\
\hline & & VMs & \\
\hline \multicolumn{4}{|c|}{$\begin{array}{l}\text { Amount of computation-intensive cloudlets } 1800 \\
\text { (including high_pay and low_pay) }\end{array}$} \\
\hline \multirow{3}{*}{ Cloudlet } & $\begin{array}{l}\text { Amount of co } \\
\text { \& high_paycl }\end{array}$ & $\begin{array}{l}\text { mputation-intensive } \\
\text { loudlets }\end{array}$ & $\begin{array}{l}180(10 \% \text { of total computation- } \\
\text { intensive cloudlets) }\end{array}$ \\
\hline & $\begin{array}{l}\text { Amount of stc } \\
\text { (including hig }\end{array}$ & $\begin{array}{l}\text { orage-requesting cloudlets } \\
\text { h_pay and low_pay) }\end{array}$ & 1200 \\
\hline & $\begin{array}{l}\text { Amount of stc } \\
\text { cloudlets }\end{array}$ & orage-intensive $\&$ high_pay & $\begin{array}{l}120(10 \% \text { of total storage- } \\
\text { requesting cloudlets) }\end{array}$ \\
\hline
\end{tabular}

Average arrival interval

Follow Poisson curve

5.2. Experimental Results and Discussion

Regardless, the exploratory results for VM3 and VM4 is immediately discussed, as a target of setting up VM3 and VM4's basically lower limit than VM1 and VM2's is to test the condition in which some Clouds requesting high capable machines can be spread to a machine with a cut down limit (VM3 and VM4 in this preliminary). In this case, the measure of Clouds requesting VM1 and VM2 outperform the most outrageous size of VM1 and VM2, keeping in mind the end goal to keep up a key separation from large holding up time before a machine with a higher limit (VM1 
and VM2 in this examination). Among the 4 VMs, VM1 is more sensible for Clouds requesting computation organization, and VM2 is more suitable for Clouds requesting limit organization, and VM3 and VM4 can give both estimations and limit advantage, yet their ability and speed for count and limit getting ready are lower than VM1 and VM2. Offensive channel identifying with cutting down level breaking point (LoT) is simply connected on attribute 1 , and the strict channel is contrasting with more raised sum edge $(\mathrm{HiT})$ is constrained on both attribute1 and property 2. For discussing the investigation comes to fruition, two conditions should be seen as VM line evaluate is under lower restrict, or VM line measure outperforms cut down the edge. We look at the results from the two points of view because: under lower edge, disagreeable filtering instrument is associated; and once cut down breaking point is accomplished, the strict isolating framework is initiated. After the filtering by VM1 and VM2, the straggling leftovers of the Clouds are directed to VM3 and VM4 to ensure all benefits in the server cultivate are utilized with no waste or waiting, and all Clouds are being taken care of to keep away from motivating customers that their requests are not served. In the tests, VM1 and VM2 is the point of convergence of trade for the execution evaluation of TSAT. In this context, their properties are more perceived from each other, and the migration of Clouds filtered through of VM1 and VM2 to VM3 and VM4 is an indispensable system of load altering, which isn't the point of convergence of this paper. Among the 3000 Clouds, 1800 Clouds have attribute1 of 'count genuine's, and the other 1200 Clouds have attribute1 of 'limit - concentrated'. For performing clear yet general examinations, we have tried 4 VMs and 3000 Clouds. The Clouds with 'attribute1=compution intensive' are picked by brutal channel for VM1, and 'property = storage_intensive' for VM2. As for channel, 'attribute1=computation_intensive \&\& attribute2=high_pay' are picked for VM1 and 'attribute1 = limit_intensive \&\& attribute2 = high_pay' for VM2. In the examinations, each cloudlet has two properties: attribute 1 and property 2.

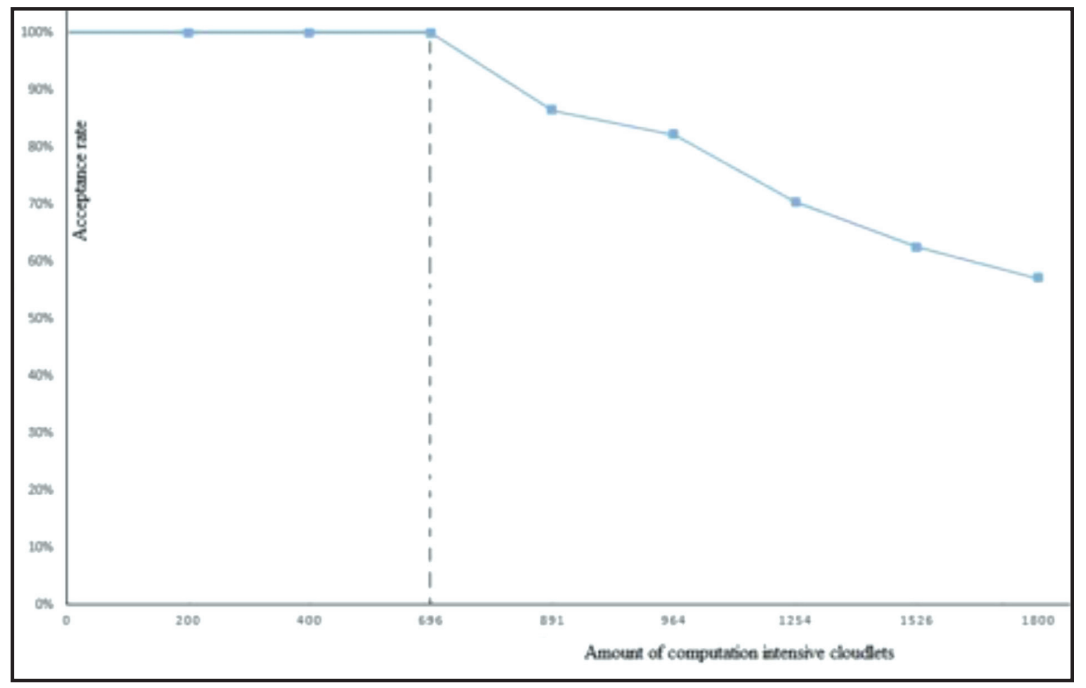

Figure 9. Overall Acceptance rate of VM1 to computation-intensive clouds 


\section{Conclusion and resolutions}

Moreover, different parameters, for instance, affirmation rate, execution time, landing between time, section speed, and taking care of speed are grasped to assess the execution of errand booking and resource circulation. When a VM's LoT is full, and hence the VM's HiT starts to work and constrains a stricter screening of characteristics on late coming properties, then the affirmation rate of the Clouds with to a significant degree one of a kind attributes (e.g., relative qualities like "high pay + count concentrated") is in like manner as high as $100 \%$. Right when the measure of coating Clouds outperforms the most extraordinary farthest point of LoT, a stricter isolating of attributes is started for the HiT of the VM to ensure the Clouds with especially new qualities (e.g., high need or urgency) can be anywise coordinated a practical resource or stick to the session. Since an errand in dispersed processing is routinely named as a cloudlet or isolated into Clouds, and a benefit is much of the time addressed by a VM (VM), so this paper based on the change of a procedure to improve the planning among Clouds and VMs.

For expressing the investigation clear, this paper develops a strategy of TSAT (Thresholds with Attributes and Amount) that can channel assorted Clouds to different fitting VMs in light of the Clouds' qualities and also the useful measure of covering Clouds before the VMs.

For breaking down the execution of TSAT, this paper proposes an enhanced TSAT technique involving a lower edge (LOT) and a higher edge (HiT), and the HiT powers a stricter screening of qualities than LoT on Clouds applying for the control of a severe VM. It is done up from the amusement happens that using the TSAT would altogether be able to improve the execution of undertaking arranging and resource distribution, particularly to the extent the perfect planning among Clouds and VMs.

- Mathematical showing can be made to characterize the coating and thresholding movement, which can be used as a summed up gadget to envision the stream of Clouds and resources, and also design the endeavor arranging and resource partition figuring.

- The affirmation rate of Clouds is affected by the transitional minute at which a VM's LOT is substantially full, and after starting of the HiT, and the transitional minute depends upon the speed of Clouds arriving at the server cultivate and likewise the speed of being set up by the VM.

- It is found that most past errand arranging and resource assignment strategies see assignments and resources as homogenous and consequently the frameworks generally base on dealing with the measure of Clouds.

- Through the composition think about on different systems for improving the idea of organization (QoS) for the impacting appropriated registering industry, this paper reasons that the execution of undertaking is arranging and resource task altogether influence the execution of all procedures.

- This methodology may in like manner conceivably avoid migration of Clouds having a place with the same colossal task beginning with one central point then onto the following center point, consequently, help achieve stick-to-session objective and in the in the meantime save the framework transmission time for stack modifying reason in dispersed registering structure. 
- The trade-off comes to be fruitful as a result of the activation of HiT. Some lately arriving Clouds that are proper for a VM may be composed to another VM, in light of the way that these Clouds don't arrange the stricter screening measures of the HiT. E.g., high pay + estimation raised. In this way are not allowed to enter the line of the sensible VM, disregarding the way that they facilitate the cruel filtering standards (e.g., figuring concentrated yet with no high pay).

Considering, the TSAT can upgrade the execution of undertaking booking and resource distribution for server homesteads, operators and customers in cloud condition. In any case, the affirmation of specially composed and late coming Clouds (e.g., high pay + count concentrated) is conceded by sorting out early coming Clouds. E.g., low pay + figuring raised. Because the unobtrusive sorted out are still allowed to enter the line due to the obstructed sanctioning of HiT which filters through unassuming composed Clouds once for the most part being incited. In layout, the going with test disclosures can reflect the change: (1) If the LoT of a VM isn't full. Then the cloudlet affirmation rate by the VM can be as high as $100 \%$, regardless of the way that the VM powers unsavory filtering of cloudlet qualities (e.g., parental attributes like "figuring heightened" and "limit concentrated"). This approach may help the progression of affirmation rate and execution time of different Clouds

and may guarantee a practical resource can be continually apportioned to a high degree one of a kind Clouds, or help a far-reaching errand containing different Clouds to cling to a corresponding resource. For assessing the execution of the TSAT strategy in a certifiable cloud condition, an open source cloud generation tool stash (CloudSim) is utilized to give an imitated cloud condition and complete the proposed TSAT, through melding different codes shaped in this paper into the Clouds. Tragically, quality - based endeavor arranging and resource divide and declare frameworks have in the field of dispersed figuring. Regardless, different future examinations are required to upgrade the TSAT; this paper prescribes the going with not all that removed future works the maker acknowledges achievable: (1) Attributes should be described entirely and representatively for the two errands and resources, as organizing depends upon the similarity of properties.

- Lessening the arrival speed or growing the best coating length of LoT, thwarted transitional minute is thwarted and extended the general affirmation rate.

- The trade-off does not mean the execution of TSAT to the extent suitability is lower than a non-channel course of action, because as far as possible are persistently endeavoring to fit Clouds to fitting VMs, this is still much better than anything unsuitable Clouds frequently control the VMs.

- As a result, the general affirmation rate is still significantly higher than a routine procedure that does not consider cloudlet characteristics.

- This process may on an elementary level upgrade the guiding of Clouds with different credits to the VMs that best fit the characteristics.

- In the TSAT framework, trademark - arranged breaking points or channels are set for the affirmation or expulsion of Clouds to the line of a VM.

- Vendor or load balancer can use historical resource status and property organizing information for early game-plan of benefit apportioning and layout change of future 
cloud devices.

- It is done up from the diversion breaks down that lessening the relative speed frustrates transitional minute and extends the general affirmation rate.

- The most outrageous coating farthest point of LoT or HiT can be expected to be continuously adaptable keeping in mind the end goal to acclimate to fluctuating sales and Clouds.

- Relative speed described as the extent of section speed to planning is gotten in this paper to fairly assess the thresholding and coating movement and pass on a theory.

- In honest to goodness cloud, in any case, different errands and machines may have distinct features or characteristics.

- Similar to the execution time decreasing came to fruition in light of the working of LoT, the execution time came to fruition due to the working of HiT is only $50-60 \%$ of an ordinary non-channel procedure under the exploratory conditions of this paper.

- Under the exploratory conditions of this paper, the ordinary execution time using TSAT is only $50-70 \%$ of a conventional non-channel methodology.

\section{Reference}

[1] Satyanarayanan, M., Bahl, V., Caceres, R., \& Davies, N. (2009). The case for vm-based cloudlets in mobile computing. IEEE pervasive Computing.

[2] Calheiros, R. N., Ranjan, R., De Rose, C. A., \& Buyya, R. (2009). Cloudsim: A novel framework for modeling and simulation of cloud computing infrastructures and services. arXiv preprint arXiv:0903.2525.

[3] Calheiros, R. N., Ranjan, R., Beloglazov, A., De Rose, C. A., \& Buyya, R. (2011). CloudSim: a toolkit for modeling and simulation of cloud computing environments and evaluation of resource provisioning algorithms. Software: Practice and experience, 41(1), 23-50.

[4] Anderson, J. (2012, February). The President's Budget: Making Cloud Computing a Priority for the Future. SafeGov. Retrieved from http://safegov.org/2012/2/16/thepresident\%E2\%80\%99sbudget-making-cloud-computing-a-priority-for-the-future

[5] Mell, P. M., \& Grance, T. (2011). The NIST definition of cloud computing. doi:10.6028/nist.sp.800-145

[6] Foster, I., Zhao, Y., Raicu, I., \& Lu, S. (2008, November). Cloud computing and grid computing 360-degree compared. In Grid Computing Environments Workshop, 2008. GCE’08 (pp. 1-10). IEEE.

[7] Kalagiakos, P., \& Karampelas, P. (2011, October). Cloud computing learning. In 2011 th International Conference on Application of Information and Communication Technologies (AICT) (pp. 1-4). IEEE.

[8] Buyya, R., Yeo, C. S., Venugopal, S., Broberg, J., \& Brandic, I. (2009). Cloud computing and emerging IT platforms: Vision, hype, and reality for delivering computing as the 5th utility. Future Generation computer systems, 25(6), 599-616.

[9] Carolan, J., Gaede, S., Baty, J., Brunette, G., Licht, A., Remmell, J., \& Weise, J. (2009). Introduction to cloud computing architecture. White Paper, 1st edn. Sun Micro Systems Inc.

[10] Zhang, Q., Cheng, L., \& Boutaba, R. (2010). Cloud computing: state-of-the-art 
and research challenges. Journal of internet services and applications, 1(1), 7-18.

[11] Rimal, B. P., Choi, E., \& Lumb, I. (2009, August). A taxonomy and survey of cloud computing systems. In Fifth International Joint Conference on INC, IMS and IDC, 2009. NCM'09 (pp. 44-51). IEEE.

[12] Crago, S., Dunn, K., Eads, P., Hochstein, L., Kang, D. I., Kang, M., Modium, D., Singh, K., Suh, J., Walters, J. P. (2011, September). Heterogeneous cloud computing. In 2011 IEEE International Conference on Cluster Computing (CLUSTER) (pp. 378385). IEEE.

[13] Brar, H. S., \& Thapar, V. An Efficient Priority Based Load Balancing Algorithm for Cloud Environment. International Journal of Computer Science Trends and Technology (IJCST)-Volume, 2, 99-103.

[14] Foley, R. D., \& McDonald, D. R. (2001). Join the shortest queue: stability and exact asymptotics. Annals of Applied Probability, 569-607.

[15] Jiang, L., Shah, D., Shin, J., \& Walrand, J. (2010). Distributed random access algorithm: scheduling and congestion control. IEEE Transactions on Information Theory, 56(12), 6182-6207.

[16] Hemamalini, M. (2012). Review on grid task scheduling in distributed heterogeneous environment. International Journal of Computer Applications, 40(2), 24-30.

[17] Mohapatra, S., Rekha, K. S., \& Mohanty, S. (2013). A comparison of four popular heuristics for load balancing of virtual machines in cloud computing. International Journal of Computer Applications, 68(6).

[18] Ming, G., \& Li, H. (2012). An improved algorithm based on max-min for cloud task scheduling. In Recent Advances in Computer Science and Information Engineering (pp. 217-223). Springer, Berlin, Heidelberg.

[19] Bhoi, U., \& Ramanuj, P. N. (2013). Enhanced max-min task scheduling algorithm in cloud computing. International Journal of Application or Innovation in Engineering and Management (IJAIEM), 2(4), 259-264.

[20] Tawfeek, M. A., El-Sisi, A., Keshk, A. E., \& Torkey, F. A. (2013, November). Cloud task scheduling based on ant colony optimization. In 2013 8th International Conference on Computer Engineering \& Systems (ICCES) (pp. 64-69). IEEE.

[21] Yadav, R. K., \& Kushwaha, V. (2014, August). An energy preserving and fault tolerant task scheduler in cloud computing. In 2014 International Conference on Advances in Engineering and Technology Research (ICAETR) (pp. 1-5). IEEE.

[22] Gahlawat, M., \& Sharma, P. (2013). Analysis and Performance Assessment of CPU Scheduling Algorithms in Cloud using CloudSim. International Journal of Applied Information Systems, 5(9), 5-8. doi:10.5120/ijais13-450970

[23] Wu, X., Deng, M., Zhang, R., Zeng, B., \& Zhou, S. (2013). A task scheduling algorithm based on QoS-driven in cloud computing. Procedia Computer Science, 17, 1162-1169.

[24] Jung, J. K., Kim, N. U., Jung, S. M., \& Chung, T. M. (2013). Improved CloudSim for simulating QoS-based cloud services. In Ubiquitous Information Technologies and Applications (pp. 537-545). Springer, Dordrecht.

[25] Gupta, G., Kumawat, V. K., Laxmi, P. R., Singh, D., Jain, V., \& Singh, R. (2014, August). A simulation of priority based earliest deadline first scheduling for cloud 
computing system. In 2014 First International Conference on Networks \& Soft Computing (ICNSC) (pp. 35-39). IEEE.

[26] Vijayalakshmi, R., \& Prathibha, S. (2013, July). A novel approach for task scheduling in cloud. In 2013 Fourth International Conference on Computing, Communications and Networking Technologies (ICCCNT) (pp. 1-5). IEEE.

[27] Ranaweera, S., \& Agrawal, D. P. (2000). A task duplication based scheduling algorithm for heterogeneous systems. In Proceedings of the 14th International Parallel and Distributed Processing Symposium, 2000. IPDPS 2000 (pp. 445-450). IEEE.

[28] Macías Lloret, M., Fitó, J. O., \& Guitart Fernández, J. (2010). Rule-based SLA management for revenue maximisation in cloud computing markets. In Proceedings of the 2010 International Conference on Network and Service Management (pp. 354357). IEEE Computer Society Publications.

[29] Chen, R., Zhang, Y., \& Zhang, D. (2013, December). A Cloud Task Scheduling Algorithm Based on Users' Satisfaction. In 2013 Fourth International Conference on Networking and Distributed Computing (pp. 1-5). IEEE.

[30] Householder, R., Arnold, S., \& Green, R. (2014, June). Simulating the effects of cloud-based oversubscription on datacenter revenues and performance in single and multi-class service levels. In Cloud Computing (CLOUD), 2014 IEEE 7th International Conference on (pp. 562-569). IEEE.

[31] Zhao, Y., Chen, L., Li, Y., \& Tian, W. (2014). Efficient task scheduling for Many Task Computing with resource attribute selection. China Communications, 11(12), 125-140.

[32] Harzog, B. (2010). Infrastructure Performance Management for Virtualized Systems. White Paper, APM Experts, 1-18.

[33] Bannerman, P. (2010). Cloud computing adoption risks: state of play. Governance, 3(16), 2-0.

[34] Nallakumar, R., \& Sruthi Priya, K. S. (2014). A survey on scheduling and the attributes of task scheduling in the cloud. Int. J. Adv. Res. Comput. Commun. Eng, 3(10), 8167-8171.

[35] Floyd, S., \& Jacobson, V. (1993). Random early detection gateways for congestion avoidance. IEEE/ACM Transactions on networking, 1(4), 397-413.

[36] Lee, G., \& Katz, R. H. (2011, June). Heterogeneity-Aware Resource Allocation and Scheduling in the Cloud. In HotCloud.

[37] Vaidehi, M., Nair, T. G., \& Suma, V. (2014). An Efficient Job Classification Technique to Enhance Scheduling in Cloud to Accelerate the Performance. In ICT and Critical Infrastructure: Proceedings of the 48th Annual Convention of Computer Society of India-Vol I (pp. 593-603). Springer, Cham.

[38] Arpaci-Dusseau, R. H., \& Arpaci-Dusseau, A. C. (2014). Operating systems: Three easy pieces (Vol. 151). Wisconsin: Arpaci-Dusseau Books.

[39] Ashraf, E., Rauf, A., \& Mahmood, K. (2012). Value based regression test case prioritization. In Proceedings of the world congress on engineering and computer science (Vol. 1, pp. 24-26).

[40] Borade, J. G., \& Khalkar, V. R. (2013). Software project effort and cost estimation techniques. International Journal of Advanced Research in Computer Science and Software Engineering, 3(8). 
[41] Barara, C., \& Maitra, S. (2012). Cloud Based Software Testing Services. International Journal of Computer Science, 1(9).

[42] Chandane, S. H., \& Bartere, M. M. (2013). New Computing Paradigm: Software Testing in Cloud, Issues, Challenges and Need of Cloud Testing in today's World. International Journal of Emerging Research in Management \&Technology, Feburary.

[43] Chetan, R., Ranjith, J., Umesh, M. \& Usha, N. (2015). Safety Platform of Traffic in Cloud Computing Environment. International Journal of Innovative Research in Computer and Communication Engineering, 3(1).

[44] Gao, J., Bai, X., \& Tsai, W. T. (2011). Cloud testing-issues, challenges, needs and practice. Software Engineering: An International Journal, 1(1), 9-23.

[45] Pundhir, Y. S. (2013). Cloud computing applications and their testing methodology. Bookman International Journal of Software Engineering, 2(1), 1-4.

[46] Hengliang, S., Changwei, Z., Tao, H., \& Yongsheng, D. (2013). Research on distributed software testing platform based on cloud resource. International Journal of Computer Science and Engineering Survey, 4(2), 17.

[47] Incki, K., Ari, I., \& Sözer, H. (2012, June). A survey of software testing in the cloud. In 2012 IEEE Sixth International Conference on Software Security and Reliability Companion (SERE-C) (pp. 18-23). IEEE.

[48] Jayashree, J.\& Vijayashree, J.(2015), Software Testing in Cloud, International Journal of Engineering Research and General Science, 3(1).

Submitted 07.07.2018 Accepted 30.10.2018 